\title{
Protective effects of dexmedetomidine on the ischemic myocardium in patients undergoing rheumatic heart valve replacement surgery
}

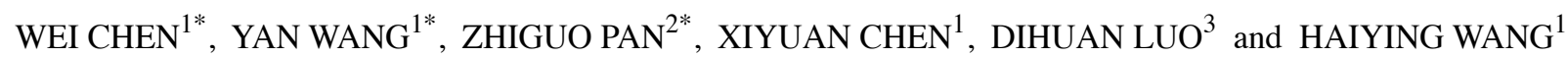 \\ ${ }^{1}$ Department of Anesthesiology, Affiliated Hospital of Zunyi Medical University, Zunyi, Guizhou 563000; \\ ${ }^{2}$ Department of Anesthesiology, Guizhou Provincial People's Hospital, Guiyang, Guizhou 550002; \\ ${ }^{3}$ Department of Anesthesiology, Affiliated Stomatological Hospital of Zunyi Medical University, \\ Zunyi, Guizhou 563000, P.R. China
}

Received January 12, 2020; Accepted May 1, 2020

DOI: $10.3892 /$ etm.2021.9844

\begin{abstract}
The aim of the present study was to compare the effects of two methods of dexmedetomidine (Dex) administration on myocardial injury, inflammation and stress in ischemic myocardium during rheumatic heart valve replacement. In total, 90 patients were included in the present study and were divided into the following three groups: i) Dex group $(1.0 \mu \mathrm{g} / \mathrm{kg}$ Dex pre-administered $10 \mathrm{~min}$ prior to anesthesia, then $0.5 \mu \mathrm{g} / \mathrm{kg} / \mathrm{h}$ Dex for maintenance); ii) Dex pre-conditioning group (Pre-Dex; $1.0 \mu \mathrm{g} / \mathrm{kg}$ Dex administered 10 min prior to anesthesia, then saline for maintenance); and iii) control group (saline $10 \mathrm{~min}$ prior to anesthesia and saline during maintenance), with 30 patients in each group. Heart rate (HR) and mean artery pressure (MAP) were recorded at eight time-points: i) T1, pre-medication; ii) $\mathrm{T} 2,10 \mathrm{~min}$ post-medication; iii) T3, immediately post-intubation; iv) T4, upon skin incision; v) T5, upon sawing the sternum; vi) T6, immediately post-cardiopulmonary bypass; vii) T7, immediately post-operation; and viii) $\mathrm{T} 8,24 \mathrm{~h}$ post-operation. The serum cardiac troponin I (cTnI), interleukin (IL)-8, IL-10 and malondialdehyde (MDA) levels were also detected at T1, T6, T7 and T8. Blood glucose levels were detected at T1, T5, T6 and T7. In comparison with the control group, patients in the Dex group exhibited a significant increase in cardiac function, as indicated by an increase in HR, MAP and IL-10 levels, and a significant decrease in cTnI, IL-8, MDA and glucose levels.
\end{abstract}

Correspondence to: Professor Haiying Wang, Department of Anesthesiology, Affiliated Hospital of Zunyi Medical University, 149 Dalian Road, Huichuan, Zunyi, Guizhou 563000, P.R. China E-mail: wanghaiting-8901@163.com

\section{${ }^{*}$ Contributed equally}

Key words: dexmedetomidine, cardiac protection, rheumatic heart valve replacement surgery, cardiac pulmonary bypass, myocardial ischemia/reperfusion injury
Both Dex perfusion and Dex preconditioning were able to reduce myocardial injury, inflammation, oxidative stress and stress response in rheumatic heart valve replacement surgery. However, Dex perfusion during the whole surgery was more effective than Dex preconditioning treatment. The study was registered with the Chinese Clinical Trial Registry (ChiCTR; no. ChiCTR-INR-17011955).

\section{Introduction}

Rheumatic heart disease frequently causes mitral stenosis and/or aortic regurgitation, which poses a serious threat to life. Heart valve replacement surgery under cardiopulmonary bypass (CPB) is a common form of surgical treatment for patients with rheumatic heart disease. However, the pathophysiological process of myocardial ischemia-reperfusion injury (MIRI) is inevitable during general anesthesia for CPB (1). MIRI is a complication of CPB and may worsen the prognosis of patients undergoing this surgical treatment. Surgeons should therefore reduce the risk of MIRI during cardiac surgery with CPB. Optimizing perioperative medications may represent an important strategy to decrease the risk of MIRI.

Previous studies have reported that ischemia preconditioning (IPC) is able to mitigate MIRI and improve cardiac function. The role of IPC in myocardial protection was first identified by Murry et al (2) using animal models and it was defined as one or more transient ischemic and reperfusion stimulations prior to ischemia. IPC is now considered to be the most effective endogenous protective measure against MIRI (3). However, the clinical application of IPC is limited due to the fact that there are no strict guidelines for the timing and duration of ischemia and the invasive nature of this complex operation. In 1997, Cope et al (4) investigated the effectiveness of drug pretreatment, which involves simulating endogenous protective factors in order to induce myocardial protection mimicking IPC. Therefore, the use of drug pretreatment to reduce MIRI has become a popular research topic.

Dexmedetomidine (Dex) is a highly selective $\alpha_{2}$-adrenoceptor agonist, the effects of which are characterized 
by sedation, analgesic and anxiolytic effects, as well as inhibition of the release of serum catecholamines (5). Dex is widely used in clinical scenarios and recent studies have indicated that the use of Dex is advantageous in reducing anesthetic requirements and enhancing hemodynamic stability following cardiac surgery (6). Dex pre-conditioning and Dex perfusion during the whole surgery have been examined in a variety of animal models of ischemia-reperfusion and have been demonstrated to protect multiple organs and provide a protective effect against MIRI (7-9). Furthermore, clinical study have suggested that Dex perfusion during the whole cardiac surgery under CPB reduces the risk of postoperative MIRI (10). However, few studies have investigated whether Dex pre-conditioning can reduce MIRI. Therefore, it has remained elusive whether Dex pre-conditioning is able to attenuate MIRI in patients undergoing rheumatic heart valve replacement surgery under CPB.

In the present study, a prospective trial was designed to investigate whether Dex pre-conditioning and Dex perfusion exert myocardial protective effects. The effects of these methods were investigated by assessing myocardial injury markers, inflammatory factors, oxidative stress and stress response in order to determine the best method of Dex administration.

\section{Materials and methods}

Study population. The present clinical study was a randomized double-blinded placebo-controlled trial that was approved by the Ethics Committee of the Affiliated Hospital of Zunyi Medical College (Zunyi, China; approval no. 8). All patients included in the present study or their relatives provided written informed consent prior to the onset of the study. In total, 105 patients were treated at the Affiliated Hospital of Zunyi Medical College (Zunyi, China) between May 2016 and August 2017. All patients were aged 18-65 years, underwent cardiac valve replacement surgery under CPB and were classified based on the American Society of Anesthesiologists (ASA) classification II or III and New York Heart Association (NYHA) classification II or III $(11,12)$, and with an estimated operation time of $\geq 2$ or $\leq 6 \mathrm{~h}$. The exclusion criteria included patients with myocardial infarction occurring in the previous month, history of heart surgery, rheumatic activity prior to surgery, heparin resistance, history of drug allergy, preoperative severe anemia, termination of anticoagulant therapy $<3$ days prior to surgery, abnormal coagulation function, severe liver and kidney damage, preoperative inflammatory disease, systemic disease with increased catecholamine secretion, relapse issues (defibrillation $>4$ times), difficulty in stopping $\mathrm{CPB}$, refusal to participate, lack of signed informed consent, withdrawal during the trial or missing data.

Patient grouping. The eligible patients were numbered sequentially according to the daily surgical schedule of the Affiliated Hospital of Zunyi Medical College (Zunyi, China) and were then randomly divided into the three study groups. Subsequently, a nurse prepared the appropriate drug and syringe, and they did not know the corresponding code. The patients and anesthesiologist were blinded to the contents of the syringe. The sample size was estimated using a statistical formula for estimating sample size for a complete randomized design with multiple sample means. The mean and standard deviation (SD) of each indicator [heart rate (HR), mean artery pressure (MAP), cardiac troponin I (cTnI), interleukin (IL)-8, IL-10, malondialdehyde (MDA) and blood glucose] in the three groups were used in the statistical formula to obtain an estimated required sample size of $\geq 27$ cases per group. In total, 90 patients were included in the trial (30 per group). These patients were blinded to their group identity. In the operating room, patients were anesthetized by the same anesthesiologist who was blinded to the patient group.

In the Dex group, patients received Dex intravenously at $1.0 \mu \mathrm{g} / \mathrm{kg} 10 \mathrm{~min}$ prior to the induction of anesthesia and then $0.5 \mu \mathrm{g} / \mathrm{kg} / \mathrm{h}$ Dex during the entire operation. In the Dex pre-conditioning group, patients received Dex intravenously at $1.0 \mu \mathrm{g} / \mathrm{kg}$ until $10 \mathrm{~min}$ prior to the induction of anesthesia and then $0.9 \%$ normal saline (at the same dose as the maintenance dose of Dex in the Dex group) during the operation. In the control group, patients were injected with $0.9 \%$ normal saline at the same doses and time-points as Dex in the Dex group (prior to the induction of anesthesia and during the operation).

Surgery. Patients who met the inclusion criteria were routinely monitored in terms of electrocardiography (ECG), pulse oxygen saturation and non-invasive blood pressure after entering the operating room. In addition, the patients were monitored using a bispectral index (BIS) monitor to assess the depth of anesthesia. MAP was monitored by cannulation of the left radial artery under local anesthesia. A double-lumen central venous catheter was inserted to monitor the central venous pressure. In addition, patients were monitored for nasopharyngeal and rectal temperature and by arterial blood gas analysis, which was also used to determine the level of blood glucose.

Anesthesia was induced with $0.01 \mathrm{mg} / \mathrm{kg}$ penehyclidine hydrochloride, $0.1 \mathrm{mg} / \mathrm{kg}$ midazolam, $1.0 \mu \mathrm{g} / \mathrm{kg}$ sufentanil, $0.15-0.3 \mathrm{mg} / \mathrm{kg}$ etomidate and $1 \mathrm{mg} / \mathrm{kg}$ rocuronium. Drug use was reduced as appropriate in patients with severe conditions (such as ASA III and NYHA III), and tracheal intubation was performed when BIS ranged between 40 and 50. After successful tracheal intubation, volume-controlled mechanical ventilation was performed and the tidal volume was adjusted to $8-10 \mathrm{ml} / \mathrm{kg}$, the inspiratory-to-respiratory ratio was maintained at $1: 1.5-2$ and the respiratory rate was maintained at $12-20$ breaths/min to ensure an end-tidal carbon dioxide partial pressure of $35-45 \mathrm{mmHg}$. Subsequently, $1-2 \mu \mathrm{g} / \mathrm{kg} / \mathrm{h}$ sufentanil, $4-6 \mathrm{mg} / \mathrm{kg} / \mathrm{h}$ propofol and intermittent intravenous injections of rocuronium and midazolam, along with compound inhalation of isoflurane (if necessary), were used to maintain the appropriate depth of anesthesia. The target range of BIS was 40-50 and the fluctuation of blood pressure was maintained at $<20 \%$.

Furthermore, the induction and maintenance of anesthesia in the three groups were using the same protocols in order to limit variability among patients. All operative procedures were performed by the same surgical team, using a CPB machine (Jostra; Sorin Group Co., Ltd.), an extracorporeal membrane oxygenator (CAPIOX RXO5, Terumo Co., Ltd.), a blood ultrafilter (SORIN DHF0.2; Sorin Group Co., Ltd.), the domestic Ningbo Filar corresponding type of arterial microthrombus filter (Ningbo Filar Medical Products Co., Ltd.) 


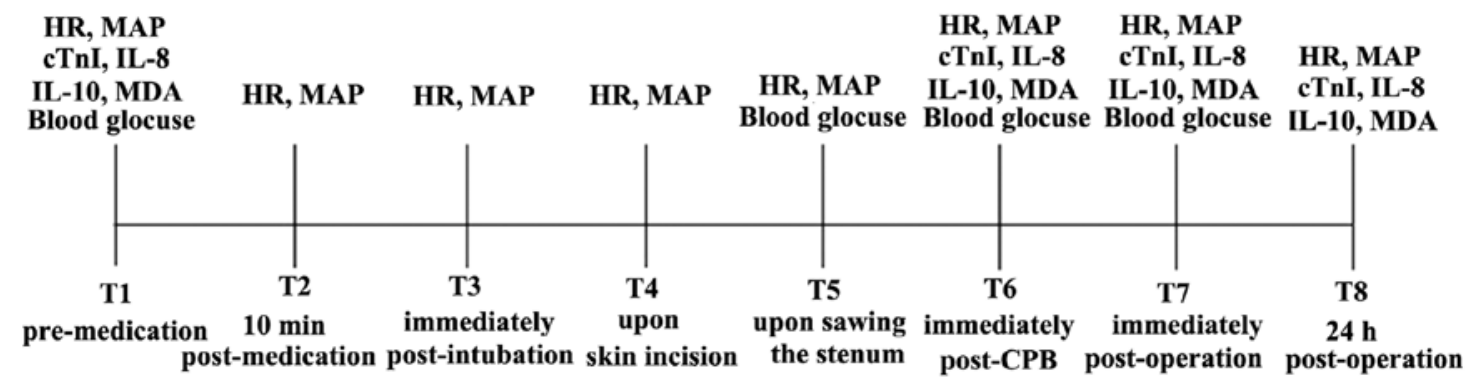

Figure 1. Timeline sketch indicating time-points and indicators detected. The time-points were as follows: i) T1, pre-medication; ii) T2, 10 min post-medication; iii) T3, immediately post-intubation; iv) T4, upon skin incision; v) T5, upon sawing the sternum; vi) T6, immediately post-CPB; vii) $\mathrm{T} 7$, immediately post-operation; and viii) T8, 24 h post-operation. HR, heart rate; MAP, mean artery pressure; cTnI, cardiac troponin I; IL, interleukin; MDA, malondialdehyde; $\mathrm{CPB}$, cardiopulmonary bypass.

and the corresponding type of non-heparin coated circulation pipeline (Ningbo Filar Medical Products Co., Ltd.). Surgery was performed under standard hypothermic CPB $\left(28-30^{\circ} \mathrm{C}\right)$, with cannulation of the superior vena cava, inferior vena cava and ascending aorta. During CPB, the following parameters were maintained: Activated clotting time of $>480 \mathrm{sec}$, hematocrit of 25-28\%, MAP of 50-80 mmHg, hemoglobin of $>80 \mathrm{~g} / 1$, $\mathrm{pH}$ of 7.35-7.45 and partial pressure of $\mathrm{CO}_{2}$ of $35-45 \mathrm{mmHg}$. Adrenaline, dopamine and nitroglycerin were intravenously pumped to maintain hemodynamic stability after stopping the $\mathrm{CPB}$.

Measurement of hemodynamic and blood parameters. HR and MAP were assessed in the three groups of patients based on ECG and invasive artery blood pressure at eight time-points: i) $\mathrm{T} 1$, pre-medication; ii) $\mathrm{T} 2,10 \mathrm{~min}$ post-medication; iii) $\mathrm{T} 3$, immediately post-intubation; iv) $\mathrm{T} 4$, upon skin incision; v) T5, upon sawing the sternum; vi) T6, immediately post-CPB; vii) T7, immediately post-operation; and viii) T8, $24 \mathrm{~h}$ post-operation. Blood samples $(5 \mathrm{ml})$ were obtained from the radial artery at four time-points (T1, T6, T7 and $\mathrm{T} 8$ ), centrifuged and then stored in a refrigerator at $4^{\circ} \mathrm{C}$. After centrifugation and storage at $-80^{\circ} \mathrm{C}$, the supernatant was collected to determine the serum levels of cTnI, IL-8, IL-10 and MDA. The concentrations of serum cTnI, IL-8 and IL-10 were assayed by ELISA using the cTnI, IL-8 and IL-10 detection kits (Beijing Biosic Biomedical Technology Co., Ltd.) according to the manufacturer's protocols. The serum level of MDA was measured using the thiobarbituric acid assay with the MDA determination kit (Beijing Solarbio Technology Co., Ltd.), according to the manufacturer's protocol. The concentration of blood glucose was detected by arterial blood gas analysis at four time-points: T1, T5, T6 and T7 (Fig. 1). All measurements were performed in duplicate.

Statistical analysis. Data analysis and statistical analyses were performed using SPSS software version 18.0 (SPSS, Inc.). Data with a normal distribution are presented as the mean \pm SD. Mixed ANOVA was used to assess the main effect of treatment, main effect of time point and the interaction between these two main effects. Afterwards, Bonferroni's correction is used for post hoc inspection. The significance of differences among the three groups was determined by one-way analysis of variance (ANOVA), when obtaining a significant result, Bonferroni's correction is used for post hoc inspection between the two groups. All tests were two-tailed. Differences in categorical variables were assessed using the $\chi^{2}$ or Fisher's exact test. All tests were two-tailed. $\mathrm{P}<0.05$ was considered to indicate statistical significance.

\section{Results}

General information of the patients and surgical conditions. A total of 105 patients were randomly allocated to the Control group ( $(n=37)$, Pre-Dex group $(n=32)$ and Dex group $(n=36)$. After randomization, four patients (three in the Control group and one in the Dex group) did not meet the inclusion criteria, since three patients in the Control group had an operation time of $>6 \mathrm{~h}$ and one patient in the Dex group had an operation time of $<2 \mathrm{~h}$. Therefore, these three patients were excluded. In addition, six patients were not followed up due to their families refused to participate after the operation and were therefore excluded due to incomplete data. Furthermore, three patients could not be followed up due to death of heart failure after operation, so they were excluded from this study. Mortality of each group [pre-Dex group, 1/32 (3.1\%), Control group, 1/37 (2.7\%) and Dex group 1/36 (2.8\%)]. Of note, the cause of death was mostly due to heart failure and were not related to Dex treatment. In addition, two patients were discharged from hospital due to serious illness and poor prognosis and were therefore excluded. Finally, 90 cases were included in the present analysis and 30 patients were allocated in each of the three groups. All patients were surgically treated and none of the patients withdrew from the study (Fig. 2). As presented in Table I, the mean age of the patients in the Pre-Dex, Dex and Control groups was $50.01 \pm 9.97,47.24 \pm 7.93$ and $51.39 \pm 6.78$ years, respectively. Male patients accounted for $87.5 \%$ of the Dex group and $76.5 \%$ of both the Pre-Dex and Control groups $(P>0.05)$. The baseline demographic characteristics were similar among the three groups $(P>0.05)$. There were no significant differences in ascending aortic cross-clamping duration, CPB duration, incidence of ventricular fibrillation after reperfusion, cardiac self-rejuvenation rate or operation duration across the three groups $(\mathrm{P}>0.05$; Table I).

Dex perfusion and Dex pre-conditioning improve heart function after $C P B$. The HR was significantly reduced in the Pre-Dex and Dex groups at T2, T3, T4 and T5 compared 
Table I. Baseline demographic characteristics, aortic cross-clamping duration, CPB duration, VFafter reperfusion, self-rejuvenation rate and operation duration in the different groups.

\begin{tabular}{|c|c|c|c|c|}
\hline Variables & Control group & Dex group & Pre-Dex group & P-value \\
\hline Age (years) & $51.39 \pm 6.78$ & $47.24 \pm 7.93$ & $50.01 \pm 9.97$ & 0.151 \\
\hline $\operatorname{Sex}(M / F)$ & $13(43) / 17(57)$ & $14(47) / 16(53)$ & $13(43) / 17(57)$ & 1.000 \\
\hline Bodyweight (kg) & $55.51 \pm 8.43$ & $53.79 \pm 8.92$ & $57.81 \pm 10.06$ & 0.239 \\
\hline NYHA (II/III) & $11(37) / 19(63)$ & $14(47) / 16(53)$ & $12(40) / 18(60)$ & 1.000 \\
\hline $\operatorname{LVEF}(\%)$ & $57.87 \pm 7.14$ & $58.60 \pm 5.54$ & $61.33 \pm 6.58$ & 0.097 \\
\hline Aortic cross-clamp duration (min) & $71.58 \pm 17.25$ & $68.52 \pm 20.65$ & $71.77 \pm 19.07$ & 0.760 \\
\hline CPB duration (min) & $106.90 \pm 20.58$ & $99.70 \pm 20.70$ & $105.80 \pm 19.27$ & 0.335 \\
\hline VF after reperfusion & $5(17)$ & $5(17)$ & $6(20)$ & 1.000 \\
\hline Self-rejuvenation rate & $25(83)$ & $25(83)$ & $24(80)$ & 1.000 \\
\hline Operation duration (min) & $249.60 \pm 42.39$ & $254.07 \pm 50.78$ & $254.87 \pm 44.00$ & 0.892 \\
\hline
\end{tabular}

Values are expressed as $\mathrm{n}(\%)$ or the mean \pm standard deviation. Pre-Dex group, Dex pre-conditioning group; Dex, dexmedetomidine; CPB, cardiopulmonary bypass; NYHA, New York Heart Association classification; LVEF, left ventricular ejection fraction; VF, ventricular fibrillation; M, male; F, female.



Figure 2. Flow diagram of the movement of patients in the present study. None of the randomized patients withdrew from the study (six were lost to follow-up). Pre-Dex group, Dex pre-conditioning group; Dex, dexmedetomidine.

with that of the Control group $(\mathrm{P}<0.00625)$, demonstrating the positive effects of DEX on myocardial oxygen consumption. However, the HR was not statistically significant among the three groups after CPB (T6, T7 and T8), suggesting that Dex had no influence on cardiac rejuvenation and HR after CPB (Fig. 3A). The HR was increased in the Pre-Dex and Dex groups after CPB (T6, T7 and T8) compared with that prior to CPB (T2-T5; P<0.00625). Furthermore, the HR was increased in control group after CPB (T6, T7) compared with that prior to $\mathrm{CPB}(\mathrm{T} 2 ; \mathrm{P}<0.00625)$.
The MAP was reduced in three groups at T4, T5, T6 and T7 compared with $\mathrm{T} 1(\mathrm{P}<0.00625)$, and there was no difference among the three groups. The MAP was reduced in the Control group at T8 compared with T1 $(\mathrm{P}<0.00625)$, while there were no differences in the Pre-Dex and Dex groups $(\mathrm{P}>0.05)$. Compared with the Control group, the MAP in Pre-Dex and Dex groups was increased at T8 $(\mathrm{P}<0.00625)$, suggesting that DEX treatment improved the MAP, thus enhancing cardiac perfusion after surgery, and had no influence on MAP during surgery (Fig. 3B). 

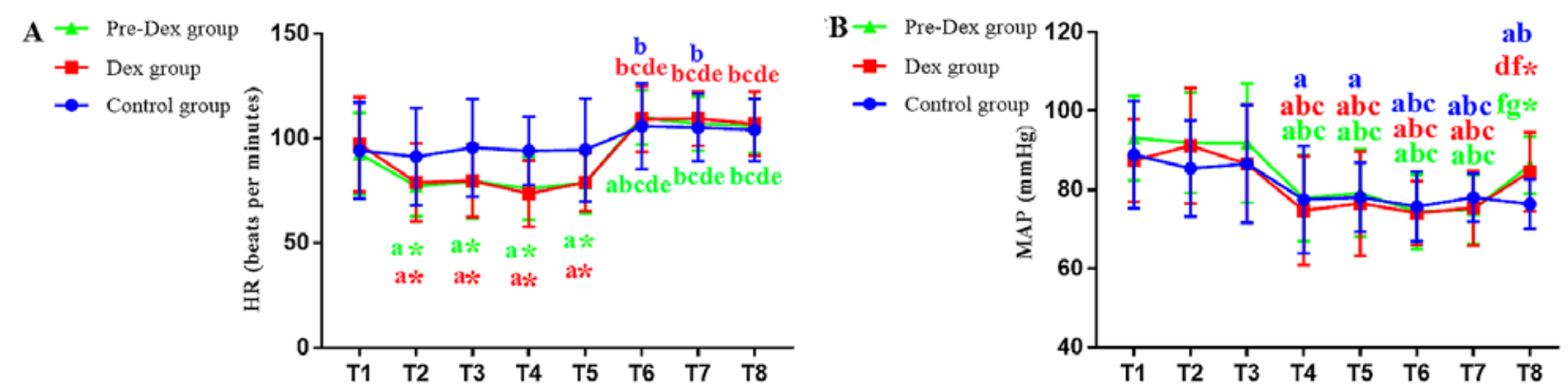

Figure 3. (A and B) Changes in HR and MAP in the three groups are shown respectively. HR and MAP in the three groups were recorded at eight time-points: i) $\mathrm{T} 1$, pre-medication; ii) $\mathrm{T} 2,10$ min post-medication; iii) $\mathrm{T} 3$, immediately post-intubation; iv) $\mathrm{T} 4$, upon skin incision; v) $\mathrm{T} 5$, upon sawing the sternum; vi) $\mathrm{T} 6$, immediately post-cardiopulmonary bypass; vii) $\mathrm{T} 7$, immediately post-operation; and viii) $\mathrm{T} 8,24 \mathrm{~h}$ post-operation. Values are expressed as the mean \pm standard deviation. ${ }^{a} \mathrm{P}<0.05$ vs. $\mathrm{T} 1,{ }^{\mathrm{b}} \mathrm{P}<0.05$ vs. $\mathrm{T} 2,{ }^{\mathrm{C}} \mathrm{P}<0.05$ vs. T3, ${ }^{\mathrm{d}} \mathrm{P}<0.05$ vs. $\mathrm{T} 4,{ }^{\mathrm{e}} \mathrm{P}<0.05$ vs. $\mathrm{T} 5,{ }^{\mathrm{f}} \mathrm{P}<0.05$ vs. $\mathrm{T} 6,{ }^{\mathrm{g}} \mathrm{P}<0.05$ vs. T7. ${ }^{*} \mathrm{P}<0.05$ vs. Control group at the same time point. Bonferroni's correction. HR, heart rate; MAP, mean artery pressure; Pre-Dex group, Dex pre-conditioning group; Dex, dexmedetomidine.

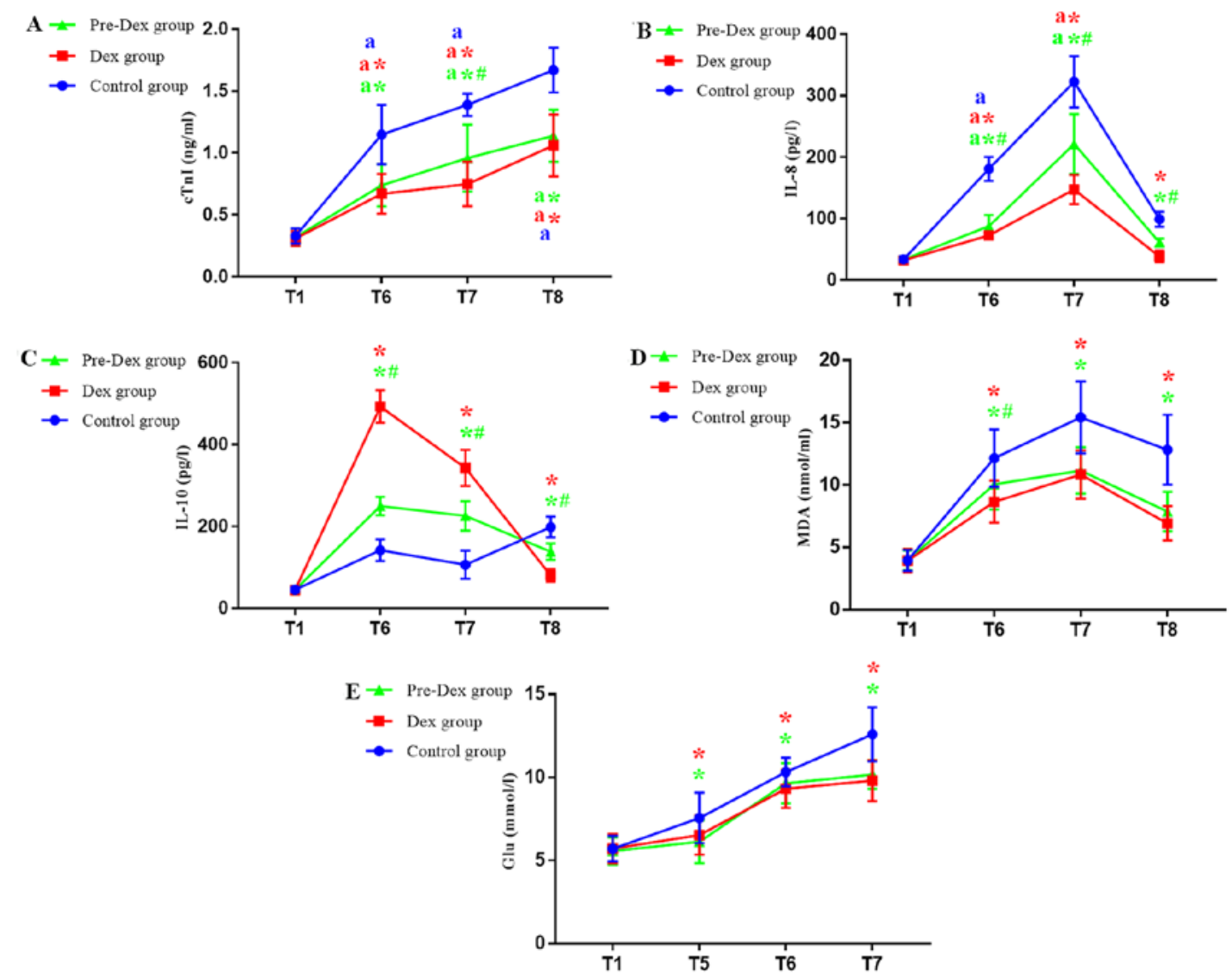

Figure 4. (A-E) Changes in the levels of cTnI, IL-8, IL-10, MDA and blood glucose in the three study groups are shown respectively. Levels of cTnI, IL-8, IL-10 and MDA in the three groups were measured at eight time-points: i) T1, pre-medication i.e. the time point before dexmedetomidine was used; ii) T2, 10 min post-medication; iii) T3, immediately post-intubation; iv) T4, upon skin incision; v) T5, upon sawing the sternum; vi) T6, immediately post-cardiopulmonary bypass; and vii) T7, immediately post-operation. Each reaction was performed three times for each patient at each time point. Values are expressed as the mean \pm standard deviation. ${ }^{\text {}} \mathrm{P}<0.05$ vs. Dex group at the same time-point; ${ }^{\mathrm{a}} \mathrm{P}<0.05 \mathrm{~T} 5-\mathrm{T} 8$ vs. T1. ${ }^{\text {P }}<0.05$ vs. the control group at the same time-point. Bonferroni's correction. cTnI, cardiac troponin I; IL, interleukin; MDA, malondialdehyde; Glu, blood glucose; Pre-Dex group, Dex pre-conditioning group; Dex, dexmedetomidine.

Dex perfusion and Dex pre-conditioning decrease the levels of myocardial injury-associated markers. In comparison with $\mathrm{T} 1$, the cTnI levels were significantly increased at T6, T7 and T8 in three groups, indicating that $\mathrm{CPB}$ induced cardiac damage $(\mathrm{P}<0.0125)$. In comparison with the Control group, cTnI levels were decreased at T6, T7 and T8 in the Pre-Dex and Dex groups $(\mathrm{P}<0.0125)$. Compared with the Pre-Dex group, the cTnI levels were decreased in the Dex group at T7 $(\mathrm{P}<0.0125)$. It was indicated that Pre-Dex and Dex treatments are able to prevent myocardial injury. In addition, the present results suggested that Dex treatment was more effective than Pre-Dex treatment (Fig. 4A).

Dex perfusion and Dex pre-conditioning regulates the levels of inflammatory factors. IL-8 gradually increased during the 
surgical procedure, reached a maximum at $\mathrm{T} 7$ and decreased at T8. During the whole procedure, in the three groups, IL-8 levels increased significantly at $\mathrm{T} 6$ and $\mathrm{T} 7$ time points compared to T1. In the control group, the level of IL-8 was significantly higher compared with that in the Pre-Dex and Dex groups at the same time point. and the IL- 8 levels in the Dex group were decreased compared with those in the Pre-Dex group $(\mathrm{P}<0.0125)$. IL-8 returned to baseline levels at $24 \mathrm{~h}$ post-operation, suggesting that $\mathrm{CPB}$ rheumatic heart valve replacement surgery stimulated the inflammatory response. However, Pre-Dex and Dex treatment downregulated the inflammatory response and Dex treatment significantly decreased the expression levels of the inflammatory marker IL-8.

IL-10 was increased at T6 in the three groups. A significant increase was identified in groups subjected to Dex treatment compared with the Control group $(\mathrm{P}<0.0125)$, suggesting that, after CPB, Pre-Dex and Dex treatment increased the release of anti-inflammatory factors and Dex treatment was more effective than Pre-Dex treatment. Compared with T6, the IL-10 levels were decreased in the three groups at the subsequent time-points, but the Pre-Dex and Dex groups presented with lower levels of IL-10 compared with those in the Control group $(\mathrm{P}<0.0125)$. At $\mathrm{T} 8$, the levels of IL-10 reached a maximum in the Control group and its levels were decreased in the Pre-Dex and Dex groups $(\mathrm{P}<0.0125$; Fig. 4B and $\mathrm{C})$.

Dex perfusion and Dex pre-conditioning suppress oxidative stress and the stress response. MDA, an indicator of oxidative stress, in the three groups, it increased from $\mathrm{T} 1$ to $\mathrm{T} 7$ and reached a maximum at the $\mathrm{T} 7$ time point before slightly decreasing at T8. In comparison with the Control group, the groups subjected to Dex treatment exhibited reduced MDA levels at T6, T7 and T8. Treatment by Dex perfusion during the whole surgery led to a more significant reduction compared with that in the Pre-Dex group at T6 ( $\mathrm{P}<0.0125$; Fig. 4D).

The glucose levels, an indicator of the stress response, increased during the whole procedure and reached a maximum at $24 \mathrm{~h}$ after surgery in all three groups. Compared with those in the Control group, the glucose levels in Pre-Dex and Dex groups were decreased $(\mathrm{P}<0.0125)$. The glucose levels were similar between the Pre-Dex and Dex groups (Fig. 4E).

\section{Discussion}

MIRI is a common pathophysiological process that occurs after cardiac surgery under CPB and involves multiple mechanisms, including cardiomyocyte apoptosis, inflammatory reactions, oxidative stress and stress responses (13-15). The pipeline for extracorporeal circulation is responsible for the process of cooling and rewarming, which causes cytokine release and promotes the occurrence and development of inflammatory and stress responses (16). Furthermore, when the blocked ascending aorta is re-opened, recanalization may promote additional production of inflammatory chemokines due to oxidative stress. The release of cytokines eventually causes damage to tissues and organs $(17,18)$. For patients with rheumatic heart disease, cardiac reserve function is reduced and hemodynamic fluctuations tend to occur during the perioperative period (19). Therefore, reducing the stress response and inflammation, and stabilizing the hemodynamics, may reduce the detrimental effects induced by MIRI.

It has been previously reported that Dex is able to inhibit the sympathetic activity of the nervous system and relieve oxidative stress reactions, inhibiting inflammation and stress response (20-22). This effect reduces the severe hemodynamic fluctuations during surgery and hemodynamic stability has a positive effect on the postoperative outcomes $(23,24)$. Dex has an HR-slowing effect prior to CPB and at the concentration used in the present study, the HR in patients in the Pre-Dex and Dex groups was lower than that in the Control group at T2, T3, T4 and T5, but only had a negligible impact on MAP. After the ascending aorta opens (recovers blood flow to the heart), it has no effect on the heart's rebound rate and the HR. Therefore, reducing myocardial oxygen consumption prior to CPB may be beneficial for MIRI. Kehlet (25) pointed out that the application of Dex during the perioperative period may have important implications for Enhanced Recovery After Surgery (ERAS). Of note, using minimally invasive techniques and fast-track cardiac anesthesia for congenital heart disease surgery was reported to promote early extubation and accelerate patient recovery, which promoted ERAS $(26,27)$.

Previous studies have indicated that Dex is able to reduce the levels of cTnI after myocardial ischemia-reperfusion, inhibit the release of the pro-inflammatory cytokine IL-8, promote the production of the anti-inflammatory cytokine IL-10 and exert antioxidant effects by reducing the level of MDA, thereby exerting cardioprotective effects $(5,22,28,29)$. The most widely used clinical markers of myocardial injury are cTnI and creatine kinase-MB. Transesophageal echocardiography cardiac function monitoring and pro-brain natriuretic peptide may also be used as indicators of myocardial function in response surgery (30). However, cTnI is a highly sensitive and specific biomarker in the diagnosis of myocardial injury in the clinic (31). In addition, Dex inhibits the release of catecholamines to reduce HR (32), which prolongs coronary perfusion and simultaneously reduces myocardial oxygen consumption, thus improving the maintenance of the myocardial oxygen supply-demand balance $(33,34)$. Dex is also able to increase the ischemic to non-ischemic myocardial blood flow ratio during myocardial ischemia in the perioperative period $(35,36)$. The effect of Dex may be associated with the activation of $\alpha_{2}$-adrenoceptors and its bidirectional regulation of the coronary artery diameter (37), thus preventing further aggravation of MIRI. The results of the present trial indicated that the levels of cTnI in the Pre-Dex and Dex groups were lower than those in the Control group at T6, T7 and T8, which means the application of DEX may reduce myocardial injury after CPB in patients with heart valve replacement surgery. This effect may be due to alterations in the timing of the release of myocardial injury markers into the blood, the peak time and the elimination half-life.

A systemic inflammatory response is able to aggravate myocardial damage (38). Previous studies have indicated that infiltration of inflammatory cells into the myocardium during MIRI leads to overproduction of reactive oxygen species, which are key in MIRI (39). IL-8 is a pro-inflammatory cytokine; mainly pro-inflammatory cells release a series of active products, leading to the occurrence of inflammatory reactions. IL-10 is mainly an anti-inflammatory cytokine 
whose major role is to inhibit inflammatory responses. The present study indicated that the levels of IL- 8 in the Pre-Dex and Dex groups were lower than those in the Control group at T6, T7 and T8, while the levels of IL-10 in the Pre-Dex and Dex groups were higher than those in the Control group at T6 and T7. Collectively, Dex administration alleviated MIRI by inhibiting the inflammatory response. In the Pre-Dex and Dex groups, IL-10 was lower compared with that in the control group at T8. This may be due to the application of DEX promoting the release of anti-inflammatory factor IL-10, inhibiting the pro-inflammatory reactions and reducing MIRI, thereby maintaining the inflammation at low levels. At T8, the level of IL-10 in the Dex group was suddenly significantly lower than that of the Pre-Dex group. This may be because the half-life of IL-10 in the body was only a few hours. The Dex group promoted IL-10 secretion during surgery. At T8 time, most were likely metabolized. In the Pre-Dex group, preconditioning induces myocardial the protection mechanism. At the T8 time point, new IL-10 is produced. Therefore, at the T8 time point, the IL-10 level of the Dex group is suddenly significantly lower than that of the Pre-Dex group. The control group demonstrated a strong inflammatory response, inducing anti-inflammatory defense mechanisms and maintaining high levels of IL-10. However, compared with those in the Dex group, the levels of IL-10 in the Pre-Dex group decreased at T6 and T7. The present results revealed that Dex perfusion is able to minimize the inflammatory response, which may protect cardiomyocytes from MIRI damage by inhibiting inflammatory factors.

MDA is an important product of lipid peroxidation in cell membranes and is associated with the level of oxygen free radicals. Due to defects in antioxidant defense mechanisms, an increased level of oxygen free radicals may promote the release of chemokines and cytokines via an inflammatory response, thus resulting in myocardial cell injury (40). The results of the present study suggested that the levels of MDA in the Pre-Dex and Dex groups were lower than those in the Control group at T6, T7 and T8. The present results suggested that the two Dex administration methods are able to attenuate the oxidative stress reaction and may serve a role in myocardial protection. However, the present results suggested that Dex administration during the whole surgery is more effective than Dex pre-conditioning. This effect may be caused by Dex-mediated improvements in antioxidant defense mechanisms, thereby reducing the production of MDA.

Previous studies have shown that reduced insulin sensitivity leads to elevated blood glucose in patients undergoing intracardiac surgery under CPB (41). The results of the present study suggested that the concentration of blood glucose in the Pre-Dex and Dex groups was lower than that in the Control group at T5, T6 and T7. This may be due to the fact that Dex application during the perioperative period may increase insulin sensitivity, thereby reducing the blood glucose levels.

In summary, the two Dex administration methods in $\mathrm{CPB}$ rheumatic heart valve replacement surgery were demonstrated to reduce myocardial injury, inhibit the release of pro-inflammatory factors, promote the release of anti-inflammatory factors, enhance the activity of antioxidant enzymes and reduce oxidative stress and stress responses. Dex perfusion during the whole surgery was more effective compared with Dex pre-conditioning. The study of perioperative myocardial protection is an important aspect of the application of ERAS in cardiac surgery. Therefore, the present study may be of great significance to the development of ERAS in cardiac surgery.

\section{Acknowledgements}

The authors would like to thank the Guizhou Anesthesia and Organ Protection Laboratory for providing the test platform. Furthermore, they thank Professor Liu Daxing, director at the Department of Cardiovascular Surgery, Affiliated Hospital of Zunyi Medical University (Zunyi, China) for his support. Specifically, Professor Liu is the chief surgeon of all patients in the present study.

\section{Funding}

The authors gratefully acknowledge the financial support by the Chinese Society of Cardiothoracic Anesthesiology Pain Branch (grant no. CSCVA-PM-2017001).

\section{Availability of data and materials}

The datasets used and/or analyzed during the current study are available from the corresponding author on reasonable request. This trial was registered at http://www.ChiCTR.org. cn under the clinical trial number ChiCTR-INR-17011955 (date of registration, July 12, 2017).

\section{Authors' contributions}

WC participated in the design and implementation of the trial. YW implemented anesthesia, including the preparation of various drugs for induction and maintenance of anesthesia, parallel arterial puncture and central venous catheterization and tracheal intubation after induction of anesthesia. ZP, DL and $\mathrm{XC}$ made substantial contributions to the acquisition of data and analysis and interpretation of the data. WC, YW, ZP and HW authenticate the raw data in this study. HW made substantial contributions to conception and design, provided guidance for the study and revised it critically for important intellectual content and gave final approval of the version to be published. All of the authors read the final manuscript and agreed to its submission.

\section{Ethics approval and consent to participate}

All of the research that was performed on the subjects followed national regulations in accordance with the relevant set of ethical principles. The Ethics Committee of the Affiliated Hospital of Zunyi Medical College (Zunyi, China; approval no. 8) approved the present study. All subjects provided written informed consent to participate in this clinical study.

\section{Patient consent for publication}

Not applicable. 


\section{Competing interests}

The authors declare that they have no competing interests.

\section{References}

1. Li M, Xu S, Geng Y, Sun L, Wang R, Yan Y, Wang H, Li Y, Yi Q, Zhang Y, et al: The protective effects of L-carnitine on myocardial ischaemia-reperfusion injury in patients with rheumatic valvular heart disease undergoing CPB surgery are associated with the suppression of NF-KB pathway and the activation of Nrf2 pathway. Clin Exp Pharmacol Physiol 46: 1001-1012, 2019.

2. Murry CE, Jennings RB and Reimer KA: Preconditioning with ischemia: A delay of lethal cell injury in ischemic myocardium. Circulation 74: 1124-1136, 1986.

3. Karwi QG, Bice JS and Baxter GF: Pre- and postconditioning the heart with hydrogen sulfide $(\mathrm{H} 2 \mathrm{~S})$ against ischemia/reperfusion injury in vivo: A systematic review and meta-analysis. Basic Res Cardiol 113: 6, 2017

4. Cope DK, Impastato WK, Cohen MV and Downey JM: Volatile anesthetics protect the ischemic rabbit myocardium from infarction. Anesthesiology 86: 699-709, 1997.

5. Wang K, Wu M, Xu J, Wu C, Zhang B, Wang G and Ma D: Effects of dexmedetomidine on perioperative stress, inflammation, and immune function: Systematic review and meta-analysis. Br J Anaesth 123: 777-794, 2019.

6. Sheikh TA, Dar BA, Akhter N and Ahmad N: A Comparative study evaluating effects of intravenous sedation by dexmedetomidine and propofol on patient hemodynamics and postoperative outcomes in cardiac surgery. Anesth Essays Res 12: 555-560, 2018.

7. Riquelme JA, Westermeier F, Hall AR, Vicencio JM, Pedrozo Z, Ibacache M, Fuenzalida B, Sobrevia L, Davidson SM, Yellon DM, et al: Dexmedetomidine protects the heart against ischemia-reperfusion injury by an endothelial eNOS/NO dependent mechanism. Pharmacol Res 103: 318-327, 2016.

8. Zhang JJ,Peng K,Zhang J,Meng XW andJiFH:Dexmedetomidine preconditioning may attenuate myocardial ischemia/reperfusion injury by down-regulating the HMGB1-TLR4-MyD88-NF-KB signaling pathway. PLoS One 12: e0172006, 2017.

9. Ren J, Li C, Liu Y, Liu H and Dong Z: Protective effect of dexmedetomidine against myocardial ischemia-reperfusion injury in rabbits. Acta Cir Bras 33: 22-30, 2018.

10. Ammar AS, Mahmoud KM, Kasemy ZA and Helwa MA: Cardiac and renal protective effects of dexmedetomidine in cardiac surgeries: A randomized controlled trial. Saudi J Anaesth 10: 395-401, 2016.

11. Dripps RD: New classification of physical status. Anesthesiology 24: 111, 1963.

12. Caraballo C, Desai NR, Mulder H, Alhanti B, Wilson FP, Fiuzat M, Felker GM, Piña IL, O'Connor CM, Lindenfeld J, et al: Clinical implications of the New York heart Association Classification. J Am Heart Assoc 8: e014240, 2019.

13. Xiong $\mathrm{W}, \mathrm{Qu} \mathrm{Y}$, Chen $\mathrm{H}$ and Qian J: Insight into long noncoding RNA-miRNA-mRNA axes in myocardial ischemia-reperfusion injury: The implications for mechanism and therapy. Epigenomics 11: 1733-1748, 2019.

14. Shen Y, Liu X, Shi J and Wu X: Involvement of Nrf2 in myocardial ischemia and reperfusion injury. Int J Biol Macromol 125: 496-502, 2019.

15. Ma C, Xu Z and Lv H: Low n-6/n-3 PUFA ratio improves inflammation and myocardial ischemic reperfusion injury. Biochem Cell Biol 97: 621-629, 2019.

16. Al-Fares A, Pettenuzzo T and Del Sorbo L: Extracorporeal life support and systemic inflammation. Intensive Care Med Exp 7 (Suppl 1): S46, 2019.

17. Natanov R, Gueler F, Falk CS, Kühn C, Maus U, Boyle EC, Siemeni T, Knoefel AK, Cebotari S, Haverich A and Madrahimov N: Blood cytokine expression correlates with early multi-organ damage in a mouse model of moderate hypothermia with circulatory arrest using cardiopulmonary bypass. PLoS One 13: e0205437, 2018.

18. O'Neill S, Ross JA, Wigmore SJ and Harrison EM: The role of heat shock protein 90 in modulating ischemia-reperfusion injury in the kidney. Expert Opin Investig Drugs 21: 1535-1548, 2012.
19. Mebazaa A, Pitsis AA, Rudiger A, Toller W, Longrois D, Ricksten SE, Bobek I, De Hert S, Wieselthaler G, Schirmer U, et al: Clinical review: Practical recommendations on the management of perioperative heart failure in cardiac surgery. Crit Care 14: 201, 2010.

20. Zhang J, Jiang H, Liu DH and Wang GN: Effects of dexmedetomidine on myocardial ischemia-reperfusion injury through PI3K-Akt-mTOR signaling pathway. Eur Rev Med Pharmacol Sci 23: 6736-6743, 2019.

21. Yu J, Yang W, Wang W, Wang Z, Pu Y, Chen H, Wang F and Qian J: Involvement of miR-665 in protection effect of dexmedetomidine against oxidative stress injury in myocardial cells via CB2 and CK1. Biomed Pharmacother 115: 108894, 2019.

22. Xu Z, Wang D, Zhou Z, Chen Q, Zhang D, Chen S, Jiang H, Jia C and Liu X: Dexmedetomidine attenuates renal and myocardial ischemia/reperfusion injury in a dose-dependent manner by inhibiting inflammatory response. Ann Clin Lab Sci 49: 31-35, 2019.

23. Vijayan NK, Talwar V and Dayal M: Comparative evaluation of the effects of pregabalin, dexmedetomidine, and their combination on the hemodynamic response and anesthetic requirements in patients undergoing laparoscopic cholecystectomy: A randomized double-blind prospective study. Anesth Essays Res 13: 515-521, 2019.

24. Ye W, Hu Y, Wu Y, Zhu Z, Jin X and Hu Z: Retrobulbar dexmedetomidine in pediatric vitreoretinal surgery eliminates the need for intraoperative fentanyl and postoperative analgesia: A randomized controlled study. Indian J Ophthalmol 67: 922-927, 2019.

25. Kehlet H: Multimodal approach to control postoperative pathophysiology and rehabilitation. Br J Anaesth 78: 606-617, 1997.

26. Hanada S, Kurosawa A, Randall B, Van Der Horst T and Ueda K: Impact of high spinal anesthesia technique on fast-track strategy in cardiac surgery: Retrospective study. Reg Anesth Pain Med: Nov 25, 2019 doi: 10.1136/rapm-2018-100213 (Epub ahead of print).

27. Zientara A, Mariotti S, Matter-Ensner S, Seifert B, Graves K, Dzemali $\mathrm{O}$ and Genoni M: Fast-track management in off-pump coronary artery bypass grafting: Dexmedetomidine provides rapid extubation and effective pain modulation. Thorac Cardiovasc Surg 67: 450-457, 2019.

28. Kim SH and Choi YS: Effects of dexmedetomidine on malondialdehyde and proinflammatory cytokines after tourniquet-induced ischemia-reperfusion injury in total knee arthroplasty. Minerva Anestesiol 86: 223-224, 2020.

29. Zhang J, Xia F, Zhao H, Peng K, Liu H, Meng X, Chen C and Ji F: Dexmedetomidine-induced cardioprotection is mediated by inhibition of high mobility group box-1 and the cholinergic anti-inflammatory pathway in myocardial ischemia-reperfusion injury. PLoS One 14: e0218726, 2019.

30. Papp A, Uusaro A, Parviainen I, Hartikainen J and Ruokonen E: Myocardial function and haemodynamics in extensive burn trauma: Evaluation by clinical signs, invasive monitoring, echocardiography and cytokine concentrations. A prospective clinical study. Acta Anaesthesiol Scand 47: 1257-1263, 2003.

31. Vaz HA, Guimaraes RB and Dutra O: Challenges in high-sensitive troponin assay interpretation for intensive therapy. Rev Bras Ter Intensiva 31: 93-105, 2019 (In Portuguese, En).

32. Yu Z, Zhang $\mathrm{P}$, Wang $\mathrm{H}$, Zhang L, Wei W, Fang W and Mu X: Effects of dexmedetomidine versus remifentanil on mothers and neonates during cesarean section under general anesthesia. Biomed Pap Med Fac Univ Palacky Olomouc Czech Repub 164: 417-424, 2020.

33. Kundra TS, Thimmarayappa A, Dhananjaya $M$ and Manjunatha N: Dexmedetomidine for prevention of skeletal muscle ischaemia-reperfusion injury in patients with chronic limb ischaemia undergoing aortobifemoral bypass surgery: A prospective double-blind randomized controlled study. Ann Card Anaesth 21: 22-25, 2018.

34. Kundra TS, Nagaraja PS, Singh NG, Dhananjaya M, Sathish N and Manjunatha N: Effect of dexmedetomidine on diseased coronary vessel diameter and myocardial protection in percutaneous coronary interventional patients. Ann Card Anaesth 19: 394-398, 2016.

35. Peng K, Qiu Y, Li J, Zhang ZC and Ji FH: Dexmedetomidine attenuates hypoxia/reoxygenation injury in primary neonatal rat cardiomyocytes. Exp Ther Med 14: 689-695, 2017. 
36. Hashemian M, Ahmadinejad M, Mohajerani SA and Mirkheshti A: Impact of dexmedetomidine on hemodynamic changes during and after coronary artery bypass grafting. Ann Card Anaesth 20: 152-157, 2017.

37. Duncan D, Sankar A, Beattie WS and Wijeysundera DN: Alpha-2 adrenergic agonists for the prevention of cardiac complications among adults undergoing surgery. Cochrane Database Syst Rev 3: CD004126, 2018.

38. Yeang C, Hasanally D, Que X, Hung MY, Stamenkovic A, Chan D, Chaudhary R, Margulets V, Edel AL, Hoshijima M, et al: Reduction of myocardial ischaemia-reperfusion injury by inactivating oxidized phospholipids. Cardiovasc Res 115: 179-189, 2019.

39. Kuznetsov AV, Javadov S, Margreiter R, Grimm M, Hagenbuchner $\mathbf{J}$ and Ausserlechner MJ: The role of mitochondria in the mechanisms of cardiac ischemia-reperfusion injury. Antioxidants (Basel) 8: 454, 2019.
40. Xu X, Rui S, Chen C, Zhang G, Li Z, Wang J, Luo Y, Zhu H and Ma X: Protective effects of astragalus polysaccharide nanoparticles on septic cardiac dysfunction through inhibition of TLR4/NF-KB signaling pathway. Int J Biol Macromol 153: 977-985, 2020

41. Zhang DS, Liang GY, Liu DX, Yu J and Wang F: Role of phosphorylated AMP-activated protein kinase (AMPK) in myocardial insulin resistance after myocardial ischemia-reperfusion during cardiopulmonary bypass surgery in dogs. Med Sci Monit 25: 4149-4158, 2019.

This work is licensed under a Creative Commons Attribution-NonCommercial-NoDerivatives 4.0 International (CC BY-NC-ND 4.0) License. 